\title{
Sequências didáticas eletrônicas para auxiliar na aprendizagem significativa em conteúdos de Patologia Humana
}

Caroline Medeiros Martins de Almeida

bio logia1@hotmail.com

002-0445-5921

Universidade Luterana do Brasil, Canoas, Rio Grande do Sul, Brasil.

Roberta Dall Agnese da Costa

$\frac{\text { r.dallagnese@gmail.com }}{0000-0002-3015-7709}$

Universidade Luterana do Brasil, Canoas, Rio Grande do Sul, Brasil.

Paulo Tadeu Campos Lopes

pclopes@ulbra.com

Universidade Luterana do Brasil, Canoas,

Rio Grande do Sul, Brasil.

\section{RESUMO}

Os alunos do curso de Educação Física possuem muitas dificuldades de apreender os conteúdos da disciplina de Patologia Humana, por esse motivo pensou-se em criar estratégias para auxiliar no seu aprendizado utilizando as tecnologias digitais. Este artigo traz resultados de uma pesquisa que teve como objetivo criar, aplicar e avaliar as possíveis contribuições de sequências didáticas eletrônicas, utilizando mapas conceituais, para auxiliar a facilitar a aprendizagem significativa nas temáticas Câncer e Fibromialgia. Foram desenvolvidas duas sequências didáticas eletrônicas num site da wikia e avaliados, mediante análise de mapas conceituais, a aquisição desses conteúdos pelos alunos. $\mathrm{Na}$ análise dos mapas conceituais, verificou-se o seu apoio na construção do conhecimento, uma vez que os alunos precisavam integrar, reconciliar e diferenciar conceitos. Esses resultados sugerem que as aulas de Patologia Humana podem ser mais interessantes, integrando-se tecnologias digitais ao cotidiano do ensino.
\end{abstract}

PALAVRAS-CHAVE: Tecnologias digitais. Aprendizagem significativa. Ensino superior. 


\section{INTRODUÇÃO}

O ensino da Patologia Humana é essencial em diversos cursos da área da saúde. Brasileiro Filho (2013) define Patologia Humana como a ciência que estuda as causas das doenças, os mecanismos que as produzem, os locais onde ocorrem e as alterações moleculares, morfológicas e funcionais que apresentam.

Os conteúdos de Patologia Humana frequentemente são vistos pelos alunos como complexos e difíceis. Essa complexidade e dificuldade geralmente estão ligadas às metodologias com que esses temas são explorados em sala de aula, uma vez que a disciplina exige conhecimentos prévios de morfologia, fisiologia e microbiologia.

Rucatti e Souza Abreu (2015) explicam que os conceitos de material didático digital e interatividade integram o contexto de novas possibilidades no tratamento da informação e na relação com o conhecimento, bem como estão atrelados à busca pela aprendizagem significativa. Neste sentido, tornam-se importantes no planejamento e execução de práticas que objetivam maior envolvimento dos estudantes. Assim, a utilização de procedimentos metodológicos diversificados que agucem os diferentes sentidos e que coloquem o sujeito da aprendizagem em contato direto com o objeto de estudo pode promover a construção do conhecimento em diferentes temáticas (ANDRÉ, 2014; VIEIRA, PEREIRA e MATOS, 2014).

Para Boghi et al. (2016) é preciso tornar a educação mais dinâmica e significativa, isto é, que faça sentido, principalmente no Ensino Superior que possui uma quantidade de trabalhos relativamente pequena quando comparada com os estudos em relação à educação básica. Segundo Padilha, Sutil e Almeida Pinto (2014) as Tecnologias Digitais podem se configurar como materiais potencialmente significativos ao mobilizar a atenção e a motivação de aprendizes.

Neste sentido, pesquisas têm sido feitas para demonstrar como as Tecnologias Digitais podem ser utilizadas para motivar e viabilizar melhores resultados no ensino e facilitar a ocorrência da aprendizagem significativa, como os estudos de Monteiro e Groenwald (2014), Neve e Melo (2014), Brão e Pereira (2015) e Costa, Almeida e Lopes (2015).

Percebendo as dificuldades dos alunos do curso de Educação Física em apreender os conteúdos de Patologia Humana, pensou-se em criar estratégias para facilitar o seu aprendizado por meio do uso das Tecnologias Digitais. Neste contexto, o objetivo deste estudo foi criar, aplicar e avaliar as possíveis contribuições de sequências didáticas eletrônicas, utilizando mapas conceituais, num site da wikia, para auxiliar a facilitar a aprendizagem significativa nas temáticas Câncer e Fibromialgia.

\section{Referencial teórico}

Atualmente a maior parte dos alunos está habituada ao mundo digital, utilizando diariamente dispositivos eletrônicos como smartphones, tablets, notebooks, tornando imprescindível a inovação das práticas pedagógicas com o intuito de acompanhar e conquistar este público acostumado a multifuncionalidade e a diferentes estímulos (RUCATTI e SOUZA-ABREU, 2015). Com esta popularização das tecnologias digitais, as formas de ensinar e aprender 
estão mudando. Aprender não é mais visto como um ato mecânico e memorístico, mas sim um processo de construção e transformação do conhecimento (GARCIA, 2013).

As tecnologias digitais apresentam-se como uma ferramenta importante para o processo de ensino e aprendizagem no sentido de proporcionar um amplo acesso às informações (ALMEIDA e ARAÚJO JR., 2015). Desta forma, ao viabilizar um planejamento de estudo para o uso das tecnologias, faz-se necessário tanto a aquisição do conhecimento técnico e científico, como domínio didático pedagógico para aplicação e avaliação desse processo por parte do professor (MARTINIANO e ROCHA, 2015).

A criação e a utilização de sequências didáticas eletrônicas baseadas nas Tecnologias Digitais, que são ferramentas do cotidiano dos alunos e de interesse dos mesmos, pode auxiliar os professores a facilitar uma aprendizagem significativa, uma vez que utilizam materiais potencialmente significativos. Sequência didática é um conjunto de atividades ordenadas, estruturadas e articuladas com o objetivo de otimizar o processo de ensino e aprendizagem para o aluno, e envolve atividades de aprendizagem e avaliação (ZABALA, 1998; DOLZ e SCHNEUWLY, 2004). Groenwald, Zoch e Homa (2009) comentam que a vantagem do uso de uma sequência didática eletrônica é a possibilidade da utilização de diferentes recursos, com padrão superior de qualidade.

Como teoria de suporte para esta proposta, optou-se pela teoria da Aprendizagem Significativa de Ausubel, que se caracteriza pela interação entre conhecimentos prévios e conhecimentos novos, e essa interação deve ser nãoliteral e não arbitrária, onde os novos conhecimentos adquirem significado para o sujeito e os conhecimentos prévios adquirem maior estabilidade cognitiva e as condições para essa aprendizagem são a predisposição para aprender, a existência de conhecimentos prévios adequados e materiais potencialmente significativos (MOREIRA, 2012; MOREIRA, 2013).

O mapa conceitual procura refletir a estrutura conceitual do conteúdo que está sendo diagramado e pode ser utilizado na obtenção de evidências da aprendizagem significativa e auxiliar na avaliação da aprendizagem (NOVAK, 2002; MOREIRA, 2005; MOREIRA, 2013). Novak (2002) define mapa conceitual como uma ferramenta de representação do conhecimento mostrando conceitos e proposições explícitas formando uma estrutura hierárquica.

Existem três características próprias dos mapas conceituais que os diferenciam de outros recursos gráficos e técnicas cognitivas: hierarquização, na qual os conceitos estão dispostos por ordem de importância ou de inclusão; seleção, em que ocorre a eleição dos termos mais significativos ou importantes de um tema, texto ou mensagem. E, por último, impacto visual, o mapa deve ser conciso e mostrar as relações entre as ideias principais de modo simples e atraente, aproveitando a notável capacidade humana para a representação (PEÑA, 2005).

A construção e reconstrução de significados pelos alunos exige que eles procurem ativamente integrar novos conhecimentos com os conhecimentos que eles já têm em sua estrutura cognitiva (NOVAK, 2002). 


\section{Metodologia}

O púbico-alvo da pesquisa foi composto por 18 alunos da disciplina de Patologia Humana do curso de Educação Física de uma universidade privada da região metropolitana de Porto Alegre, RS.

A elaboração do instrumento de pesquisa envolveu as seguintes fases: a) escolha das ferramentas tecnológicas a serem utilizadas nas sequências didáticas eletrônicas; b) pesquisa bibliográfica sobre as temáticas Câncer e Fibromialgia; c) criação dos materiais de estudo com as temáticas Câncer e Fibromialgia, englobando textos com resumo da matéria, jogos, questionários e atividades avaliativas utilizando mapas conceituais; d) criação dos roteiros de aula com a explicação do passo a passo das atividades.

A sequência didática eletrônica sobre a temática Câncer foi composta por um material de estudo com textos e figuras que ilustram os textos, dois jogos didáticos chamados "Quiz do Câncer" e "Enigma do Câncer", um questionário com questões de múltipla escolha e uma atividade avaliativa através da criação de um mapa conceitual (Figura 1). O material de estudo foi elaborado por meio de adaptações dos trabalhos de Brasileiro Filho (2013) e Contran, Kumar e Colins (2000) com o objetivo de explicar, conceituar e exemplificar o conteúdo.

Figura 1 - Captura de tela do material de estudo de Câncer.

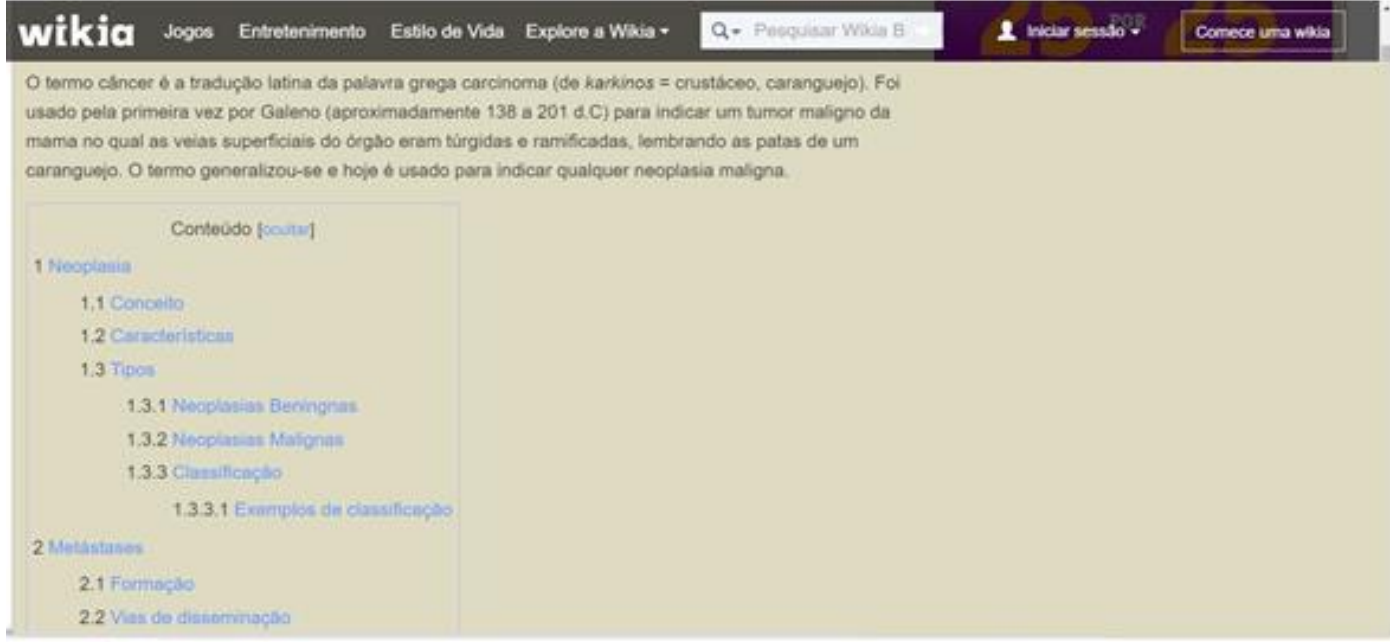

(Fonte: pt-br.biopatologia.wikia.com/wiki/Câncer)

A sequência didática sobre a temática Fibromialgia foi composta por um material de estudo com textos e figuras que ilustram os textos, um jogo didático chamado "Enigma da Fibromialgia", um questionário com questões de múltipla escolha e uma atividade avaliativa através da criação de um mapa conceitual (Figura 2). O material de estudo foi elaborado por meio de adaptações dos trabalhos de Helfenstein Junior, Goldenfum e Siena (2012) e Ferreira, Martinho e Tavares (2014) com o objetivo de explicar, conceituar e exemplificar o conteúdo. 
Figura 2 - Captura de tela do material de estudo de Fibromialgia.

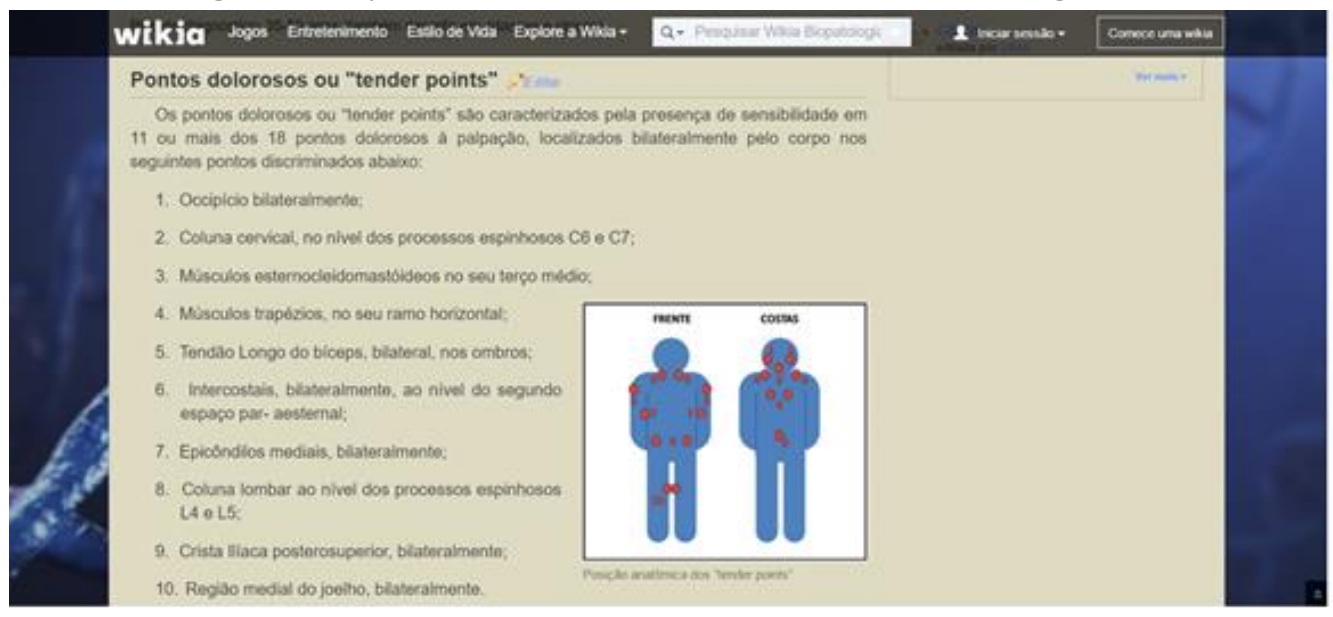

(Fonte: pt-br.biopatologia.wikia.com/wiki/Fibromialgia)

As sequências didáticas estão inseridas na página inicial da wikia que tem o nome de Biopatologia e cada atividade possui um link que leva ao material de estudo. Esse material está disponível no endereço http://ptbr.biopatologia.wikia.com.

As ferramentas tecnológicas utilizadas foram: Wikia, Hot Potatoes, LucidChart, E-mail e Formulários Google.

Wikia foi o site escolhido para ser o Ambiente Virtual de Aprendizagem da sequência didática eletrônica, pois permite que o aluno acesse o conteúdo de qualquer lugar com smartphone, tablet, notebook. Ele é um web site que geralmente permite sua alteração por qualquer pessoa que tenha um navegador de internet e possa acessar a página da wikia. A wikia utiliza um código fácil de editar que permite que se utilize textos, links e imagens sem a necessidade de aprendizado de códigos de programação, como HTML.

Hot Potatoes foi utilizado para criar os jogos e auxiliar a tornar a atividade lúdica. Ele é um software educacional canadense utilizado para criar exercícios sob a forma de objetos digitais para publicação na Web. Os jogos escolhidos foram JCloze, que cria exercícios de preenchimento de lacunas e JQuiz, que cria exercícios de escolha múltipla.

LucidChart foi utilizado para os alunos criarem um mapa conceitual do conteúdo. Trata-se de um software de diagramação baseado na Web que permite aos usuários colaborar e trabalhar em conjunto em tempo real para criar mapas conceituais, fluxogramas, organogramas, mapas mentais, e muitos outros tipos de diagramas. Construído sobre padrões da Web, como HTML5 e JavaScript, pode ser usado em todos os navegadores modernos, como Google Chrome, Firefox, Safari e Internet Explorer.

E-mail foi utilizado para os alunos enviarem os mapas conceituais, ele é um método que permite compor, enviar e receber mensagens através de sistemas eletrônicos de comunicação.

Os Formulários Google, disponíveis no Google Drive, foram usados para criar pesquisas ou votações, preparar testes para alunos, bem como coletar outras 
informações de forma simples e rápida. É possível criar um formulário a partir do Drive ou de qualquer planilha já existente e registrar as respostas nesse formulário.

As sequências didáticas eletrônicas foram criadas para os alunos estudarem as temáticas Câncer e Fibromialgia de forma diferenciada através de uma aprendizagem online, uma vez que estes ainda não haviam estudado sobre estes conteúdos durante as aulas de Patologia Humana.

A sequência didática eletrônica sobre a temática Câncer foi aplicada de modo presencial no laboratório de informática e ocorreu em duas etapas: a) a primeira etapa envolveu a explicação das atividades e a entrega de um roteiro de aula com o passo a passo das atividades. Após, iniciou-se a aplicação da primeira parte da sequência didática eletrônica em que os alunos estudaram sobre a temática Câncer, através do material de estudo disponibilizado e dos jogos educativos; b) a segunda etapa envolveu a realização da atividade avaliativa: primeiro ocorreu a explicação do conceito de mapas conceituais, a explicação de como desenhá-los e quais as funcionalidades do software LucidChart. Posteriormente, os alunos foram convidados a desenhar um mapa conceitual que expressasse o que eles sabiam sobre o conteúdo estudado.

A sequência didática eletrônica sobre a temática Fibromialgia foi aplicada em duas etapas: a) a primeira etapa foi presencial e envolveu a explicação de como realizar as atividades da sequência didática e a entrega de um roteiro de aula com o passo a passo das atividades; b) na segunda etapa, à distância, foi aplicada a sequência didática eletrônica, onde os alunos tiveram uma semana para ler o material de estudos e realizar a atividade avaliativa de criar um mapa conceitual sobre o conteúdo estudado.

Após a aplicação dos instrumentos, foram realizadas a análise dos mapas conceituais como elemento sinalizador da aprendizagem significativa. Para análise dos mapas conceituais produzidos pelos alunos foram adaptadas no presente estudo categorias desenvolvidas por Novak e Gowin (1996), Novak (2002), Peña (2005) e Ruiz-Moreno et al. (2007) (Quadro 1).

Quadro 1- Demonstrativo das categorias utilizadas para a análise dos mapas conceituais.

\begin{tabular}{|l|l|}
\hline \multicolumn{1}{|c|}{ Categoria } & \multicolumn{1}{c|}{ Descrição da categoria } \\
\hline 1- Estrutura & - se o mapa está estruturado com os conectores; \\
\hline 2- Hierarquização & $\begin{array}{l}\text { - forma de disposição dos conceitos, dos mais gerais e inclusivos para } \\
\text { os mais específicos e menos inclusivos; }\end{array}$ \\
\hline $\begin{array}{l}\text { 3- Ligações entre as } \\
\text { proposições }\end{array}$ & $\begin{array}{l}\text { - existência de ligações cruzadas entre ramos distintos da hierarquia } \\
\text { conceitual. }\end{array}$ \\
\hline
\end{tabular}




\section{Resultados e discussão}

Análise dos mapas conceituais da temática Câncer

Na categoria 1- estrutura, que verifica se o mapa está estruturado com os conectores, $28 \%$ dos mapas não estavam estruturados de acordo com as especificações da categoria, pois não possuíam conectores entre os conceitos; $44 \%$ atendiam parcialmente as especificações da categoria, faltando alguns conectores entre os conceitos e $28 \%$ atendiam as especificações da categoria, com os conectores entre os conceitos. A verificação da estrutura do mapa conceitual é importante para ele não ser confundido com mapa mental, pois, segundo Hilger e Griebeler (2013), os mapas mentais permitem ao sujeito expressar-se livremente e, portanto, influências externas na formação dos subsunçores poderiam emergir. Por outro lado, em mapas conceituais essas características são suprimidas devido à própria estrutura dos mapas, que reflete a captação de significados referentes ao conteúdo específico tratado.

$\mathrm{Na}$ categoria 2- hierarquização, que verifica a forma de disposição dos conceitos, dos mais gerais e inclusivos para os mais específicos e menos inclusivos, $33 \%$ dos mapas não estavam de acordo com as especificações da categoria, pois os conceitos estavam dispostos de forma aleatória e $67 \%$ atendiam parcialmente as especificações da categoria, expressando hierarquização e demonstrando alguns níveis de progressão entre os conceitos. A falta de hierarquia falha para indicar que os conceitos são mais inclusivos, ou mais salientes para um dado contexto para o qual a estrutura de conhecimento é para ser aplicada (NOVAK, 2002). Novak e Gowin (1996) comentam que o grau de hierarquização presente num mapa conceitual pode ser tomado como um indício da diferenciação progressiva ou da reconciliação integrativa. Diferenciação progressiva em que o subsunçor vai se tornando cada vez mais elaborado, mais diferenciado, mais capaz de servir de âncora para a atribuição de significados a novos conhecimentos, que ocorre no curso da aprendizagem significativa (MOREIRA, 2005).

$\mathrm{Na}$ categoria 3- ligações entre as proposições, que verifica a existência de ligações cruzadas entre ramos distintos da hierarquia conceitual, $28 \%$ dos mapas não estavam de acordo com as especificações da categoria, pois os conceitos estavam dispostos de forma aleatória e com pouca interação com o tema principal, $55 \%$ atendiam parcialmente as especificações da categoria, inserindo algumas associações entre ramos distintos e $17 \%$ atendiam as especificações da categoria, inserindo associações bem organizadas e com modos diferenciados de abordagem entre ramos distintos. Segundo Novak e Cañas (2008) na criação de novos conhecimentos, ligações cruzadas representam muitas vezes saltos criativos e ajudam a ver como um conceito em um domínio do conhecimento representado no mapa é relacionado a um conceito em outro domínio mostrado no mapa.

Os mapas conceituais constituem um recurso de aprendizagem significativa, capaz de auxiliar o estudante a integrar novos conceitos a conceitos pré-existentes, estabelecendo estruturas hierárquicas a partir de conceitos mais abrangentes que vão sendo progressivamente relacionados a outros mais específicos e como um meio de avaliação do aprendizado, capaz de evidenciar onde ocorreu aprendizagem satisfatória de conceitos e onde é necessário trabalhar para melhorar a compreensão (OLIVEIRA, SANTOS e ALMEIDA, 2015). 
Na categoria 1- estrutura, que verifica se o mapa está estruturado com os conectores, $25 \%$ dos mapas não estavam estruturados de acordo com as especificações da categoria, pois não possuíam conectores entre os conceitos; $50 \%$ atendiam parcialmente as especificações da categoria, faltando alguns conectores entre os conceitos e $25 \%$ atendiam as especificações da categoria, com os conectores entre os conceitos. Segundo Novak (2002) os mapas conceituais que não especificam as ligações entre os conceitos falha, pois não formam proposições que são os elementos essenciais para representar o conhecimento.

Na categoria 2- hierarquização, que verifica a forma de disposição dos conceitos, dos mais gerais e inclusivos para os mais específicos e menos inclusivos, $25 \%$ dos mapas não estavam de acordo com as especificações da categoria, pois os conceitos estavam dispostos de forma aleatória, $50 \%$ atendiam parcialmente as especificações da categoria, expressando hierarquização e demonstrando alguns níveis de progressão entre os conceitos e $25 \%$ atendiam as especificações da categoria, expressando hierarquização e demonstrando níveis de progressão entre os conceitos. Para Ferrão e Manrique (2014) observar se o autor de um dado mapa dispôs os conceitos de forma hierárquica pode ser utilizado como um indicativo do nível de complexidade e profundidade do conhecimento que o autor do mapa possui e deixou transparecer ao expressá-lo na representação.

$\mathrm{Na}$ categoria 3- ligações entre as proposições, que verifica a existência de ligações cruzadas entre ramos distintos da hierarquia conceitual, $25 \%$ dos mapas não estavam de acordo com as especificações da categoria, pois os conceitos estavam dispostos de forma aleatória e com pouca interação com o tema principal, $50 \%$ atendiam parcialmente as especificações da categoria, inserindo algumas associações entre ramos distintos e $25 \%$ atendiam as especificações da categoria, inserindo associações bem organizadas e com modos diferenciados de abordagem entre ramos distintos. Segundo Oliveira, Santos e Almeida (2015) a preocupação na construção de mapas cada vez mais complexos do ponto de vista das proposições e bonitos esteticamente também pode atuar como motivador para aprender, um aspecto essencial para que se obtenha uma aprendizagem significativa.

Moreira (2005) explica que na medida em que os alunos utilizam mapas conceituais para integrar, reconciliar e diferenciar conceitos, eles estão utilizando o mapeamento conceitual como um recurso de aprendizagem.

Por meio da análise dos mapas conceituais, verificou-se o seu apoio na construção do conhecimento, uma vez que os alunos precisam rever os conteúdos das sequências didáticas eletrônicas e atribuir diferentes significados e novas relações entre eles. Segundo Moreira (2005), não existe mapa conceitual "correto" de um certo conteúdo, o que o aluno apresenta é o seu mapa e o importante não é se esse mapa está certo ou não, mas sim se ele dá evidências de que o aluno está aprendendo significativamente o conteúdo.

Percebemos com os resultados obtidos que as sequências didáticas eletrônicas podem auxiliar no estudo dos conteúdos de Patologia Humana e facilitar a aprendizagem significativa. Boghi et al. (2016) comentam que caso os alunos tivessem aulas somente teóricas, possivelmente poderiam aumentar a quantidade de conceitos nos seus mapas, porém é possível que não demonstrassem o mesmo interesse em prosseguir no conhecimento do assunto. 


\section{Conclusões}

A pesquisa apresentou uma reflexão sobre o uso de mapas conceituais no Ensino Superior, como um instrumento de apoio na construção do conhecimento, uma vez que os alunos precisam rever o conteúdo e atribuir diferentes significados e novas relações entre eles. Durante o experimento, observou-se que essa pesquisa alcançou os objetivos propostos de criar, aplicar e avaliar uma sequência didática eletrônica com os conteúdos de Câncer e Fibromialgia, verificando a sua eficácia para facilitar a ocorrência da aprendizagem significativa, por meio do material de estudo disponível.

Esses resultados sugerem que as aulas de Patologia Humana podem ser mais interessantes, integrando-se tecnologias digitais ao cotidiano do ensino e podem contribuir para uma aprendizagem ainda mais efetiva, evidenciando que cada vez se torna mais necessário que os professores criem situações para proporcionar uma forma de facilitar a aprendizagem significativa.

Assim sendo, entende-se que atividades utilizando as tecnologias digitais deveriam merecer um espaço e um tempo maior na prática pedagógica cotidiana dos professores. Porém, ressalta-se que estas atividades não irão substituir o método de ensino tradicional, o que se espera é que elas sejam utilizadas como elementos complementares, de apoio ao processo ensino aprendizagem dos conteúdos a ensinar ou já ensinados. 


\title{
Electronic didactic sequences to assist in meaningful learning of human pathology contents
}

\begin{abstract}
Physical Education undergraduates face several difficulties to learn Human Pathology contents. For this reason, strategies based on digital technologies have been developed to help learning. This study presents the results of a research that developed, applied, and evaluated the possible contributions of electronic didactic sequences using conceptual maps as tools to facilitate the meaningful learning of the topics Cancer and Fibromyalgia. Two electronic didactic sequences were developed in one wikia website and evaluated based on the analysis of conceptual maps, on the learning of these contents by students. In the analysis of conceptual maps, it was their support in the construction of knowledge, as students needed to integrate, reconcile and differentiate concepts. These results suggest that Human Pathology classes may be more interesting, embedding digital technologies to the teaching activities.
\end{abstract}

KEYWORDS: Digital technologies. Meaningful learning. Higher education. 


\section{AGRADECIMENTOS E APOIOS}

Os autores agradecem à Coordenação de Aperfeiçoamento de Pessoal de Nível Superior (CAPES) pelas bolsas de estudo.

\section{NOTAS}

\section{REFERÊNCIAS}

ALMEIDA, R. R.; ARAÚJO JR., C. F. Atividades de ensino e aprendizagem de genética com o uso do tablet. Revista Produção Discente e Educação Matemática, São Paulo, v.4, n.1, p. 79-90, 2015.

ANDRÉ, B. P. O lugar da didática no ambiente virtual de aprendizagem. Revista Brasileira de Ensino de Ciência e Tecnologia, v. 7, n.3, p. 63-77, 2014.

BOGHI, C.; SHITSUKA, D. M.; RISEMBERG, R. I. C. S.; SHITSUKA, R. Estudo de caso de emprego de metodologias ativas no ensino de conceitos tecnológicos. Revista Associação Brasileira de Tecnologia Educacional, v. 31, p. 19-32, 2016.

BRÃO, A. F. S.; PEREIRA, A. M. B. Biotecnétika: possibilidades do jogo no ensino de genética. Revista Electrónica de Enseñanza de las Ciencias, v.14, n.1, p. 55-76, 2015.

BRASILEIRO FILHO, G. Bogliolo: Patologia geral. 5. ed. Rio de Janeiro: Guanabara Koogan, 2013. 312 p.

CONTRAN, R.; KUMAR, V.; COLINS, T. Robbins: Patologia estrutural e funcional. (6a ed.). Rio de Janeiro: Guanabara Koogan, 2000.

COSTA, R. D. A.; ALMEIDA, C. M. M.; LOPES, P. T. C. Avaliando um Ambiente Virtual de Aprendizagem para as aulas de Ciências no nono ano a partir de percepções dos alunos. Revista Brasileira de Ensino de Ciências e Tecnologia. Paraná. v.8, n.1, p.1-16, 2015.

DOLZ, J.; SCHNEUWLY, B. Gêneros orais e escritos na escola. Campinas: Mercado das Letras, 2004.

FERRÃO, N. S.; MANRIQUE, A. L. O uso de mapas conceituais como element sinalizador da aprendizagem significativa em cálculo. Investigações em Ensino de 
FERREIRA, G.; MARTINHO, U. G.; TAVARES, M. C. G. C. F. Fibromialgia e atividade física: reflexão a partir de uma revisão bibliográfica. Salusvita, Bauru, v. 33, n. 3, p. 433-446, 2014.

GARCIA, F. W. A importância do uso das tecnologias no processo de ensinoaprendizagem. Educação a distância, Batatais, v.3, n.1, p.25-48, 2013.

GROENWALD, C. L. O.; ZOCH, L.; HOMA, A. I. R. Sequência didática com análise combinatória no padrão SCORM. Bolema, v. 22, n. 34, p. 27-56, 2009.

HELFENSTEIN JUNIOR, M.; GOLDENFUM, M. A.; SIENA, C.A.F. Fibromialgia: aspectos clínicos e ocupacionais. Revista Associação Médica Brasileira, São Paulo, v.58, p. 358-365, 2012.

HILGER, T. R.; GRIEBELER, A. Uma proposta de unidade de ensino potencialmente significativo utilizando mapas conceituais. Investigações em Ensino de Ciências, v.18, n.1, p. 199-213, 2013.

MARTINIANO,E.; ROCHA, Z. F. D. C. O Uso do ambiente virtual de ensino e aprendizagem na disciplina de biologia. Revista Tecnologias na Educação, v.7, n. 13, p. 1-10, 2015.

MONTEIRO, A. B.; GROENWALD, C. L. O. Sequência didática eletrônica de frações: uma proposta para a recuperação paralela do Ensino Fundamental. Vidya, v.34, n. 1, p.61-84, 2014.

MOREIRA, M. A. Mapas conceituais e aprendizagem significativa. Revista Chilena de Educação Científica, v.4, n.2, p. 38-44, 2005.

MOREIRA, M. A. Al final qué es aprendizaje significativo? Revista Qurriculum, La Laguna, v. 25, p.29-56, 2012.

MOREIRA, M. A. Aprendizagem significativa em mapas conceituais. Textos de apoio ao professor de física, do PPGEnFis/IF-UFRGS, v. 24, n. 6, p. 1-49, 2013.

NEVE, B. G. B.; MELO, R. S. O Universo no bolso: tecnologias móveis de apoio didático-pedagógico para o ensino da Astronomia. Revista Eletrônica Novas Tecnologias na Educação, v. 12, n. 1, p. 1-10, 2014.

NOVAK, J. D. Meaningful learning: The essential factor for conceptual change in 
NOVAK, J. D.; CAÑAS, A. J. The theory underlying concept maps and how to construct and use them. Flórida, 2008. Disponível em: <

http://cmap.ihmc.us/docs/theory-of-concept-maps >. Acesso em: 02. abr. 2016.

NOVAK, J. D; GOWIN, D. B. Aprendendo a aprender. Lisboa: Plátano Edições Técnicas, 1996.

OLIVEIRA, G. C. D.; SANTOS, P. M. L. S.; ALMEIDA, R. S. Avaliação de conceitos de termodinâmica clássica através de mapas conceituais. Revista Brasileira de Ensino de Ciências e Tecnologia, v. 8, n. 4, p. 169-187, 2015.

PADILHA, A. S. C.; SUTIL, N.; ALMEIDA PINTO, Â. E. Tecnologias de Informação e Comunicação e aprendizagem significativa: perspectivas de professores de Ciências. Revista Tecnologias na Educação, v. 6, n. 11, p. 1-12, 2014.

PEÑA, A. Mapas Conceituais - uma técnica para aprender. São Paulo: Loyola, 2005.

RUCATTI, L. G.; SOUZA ABREU, C. BAAS: uma plataforma online para apoio à leitura e aprendizagem. Revista Eletrônica Novas Tecnologias na Educação, Porto Alegre, v. 13, n. 1, p.1-10, 2015.

RUIZ-MORENO, L.; SONZOGNO, M. C.; BATISTA, S. H.; BATISTA, N. A. Mapa conceitual: ensaiando critérios de análise. Ciência \& Educação, v. 13, n. 3, p. 453463, 2007.

VIEIRA, G. Q.; PEREIRA, L. P.; MATOS, W. R. Avaliação de espaços não formais de educação para o ensino de ciências: estudo de caso do museu Ciência e Vida, Duque de Caxias, RJ. Almanaque Multidisciplinar de Pesquisa, v. 1, n. 2, 2014.

ZABALA, A. A Prática educativa: como ensinar. Porto Alegre: Artmed, 1998. 
Recebido: 04 mai. 2016

Aprovado: 24 mai. 2016

DOI: http://dx.doi.org/10.3895/rbect.v9n2.3960

Como citar: ALMEIDA, C. M. M. DE; COSTA, R. D. A.; LOPES, P. T. C. Sequências didáticas eletrônicas

para auxiliar na aprendizagem significativa em conteúdos de Patologia Humana. Revista Brasileira de Ensino de Ciência e Tecnologia, v. 9, n. 2, 2016. Disponível em:

<https://periodicos.utfpr.edu.br/rbect/article/view/3960>. Acesso em: 8/11/2016.

\section{Correspondência:}

Av Farroupilha, 8001, 92425-900 - Canoas - Rio Grande do Sul.

Direito autoral: Este artigo está licenciado sob os termos da Licença Creative Commons-Atribuição 4.0 Internacional.

(c) (i) 